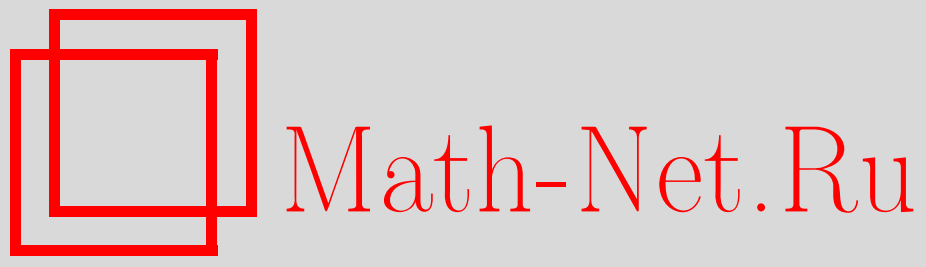

B. А. Зорич, B. М. Кесельман, Изопериметрическое неравенство на многообразиях конформногиперболического типа, Функи. анализ и его прил., 2001, том 35, выпуск 2, 12-23

DOI: https://doi.org/10.4213/faa242

Использование Общероссийского математического портала MathNet.Ru подразумевает, что вы прочитали и согласны с пользовательским соглашением

http://www . mathnet.ru/rus/agreement

Параметры загрузки:

IP : 54.198 .64 .247

26 апреля 2023 г., 11:31:45

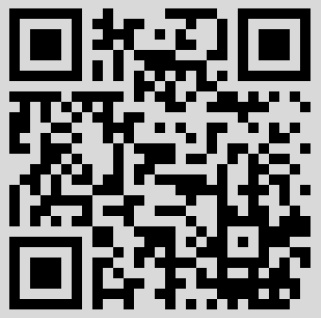




\title{
Изопериметрическое неравенство на многообразиях конформно-гиперболического типа*
}

\author{
(C) 2001. В. А. ЗОРИЧ, В. М. КЕСЕЛЬМАН
}

\section{§1. Введение}

Как известно, изопериметрическое неравенство - это соотношение вида

$$
P(V(D)) \leqslant S(\partial D)
$$

между объемом $V(D)$ области $D$ и площадью $S(\partial D)$ ее границы, где $P-$ функция, называемая изопериметрической функцией пространства. Конечно, интерес представляет только нетривиальная, а чаще всего, даже максимальная изопериметрическая функция. Например, для евклидова $n$-мерного пространства $\mathbb{R}^{n}$ таковой, с точностью до множителя, является функция $P(x)=x^{(n-1) / n}$, а для пространства Лобачевского $\mathbb{H}^{n}-$ линейная функция $P(x)=x$.

Подобно пространствам Евклида и Лобачевского, произвольные открытые римановы (и даже субримановы) многообразия можно конформно-инвариантно разделить на два класса в соответствии с тем, какова конформная емкость абсолюта (бесконечности) этого многообразия (см. $[1,2])$. Если эта емкость нулевая (как у пространства Евклида), многообразие относится к конформно-параболическому типу; если эта емкость положительна (как в случае пространства Лобачевского), многообразие относится к конформно-гиперболическому типу.

Конформная замена метрики (что на физическом языке означает калибровочное преобразование, связанное с произволом в выборе местных масштабов) не меняет конформного типа многообразия, но меняет метрические соотношения на нем.

Нас интересует канонический вид изопериметрического соотношения в конформном классе метрики многообразия, точнее, вопрос о том, к какому каноническому виду приводится изопериметрическое неравенство конформными заменами метрики многообразия.

Результаты, полученные нами в [1], позволили высказать следующую рабочую гипотезу: на любом многообразии конформно-гиперболического типа максимальная изопериметрическая функция приводится к тому же линейному виду $P(x)=x$, что и в пространстве Лобачевского, а на любом $n$-мерном многообразии конформно-параболического типа - к тому же виду $P(x)=x^{(n-1) / n}$, что и в пространстве Евклида.

В этой статье мы рассмотрим первую часть этой гипотезы и дадим положительный ответ на вопрос, поставленный в работе [3].

Часть результатов, которые мы даем здесь с доказательствами, была ранее анонсирована в [4].

*Работа выполнена при поддержке РФФИ, проект 99-01-01179. 
Напомним точное определение конформного типа многообразия. Пусть $G-$ область на открытом $n$-мерном римановом многообразии $(M, g)$, а $C$ - отличный от точки континуум (связный компакт) в $G$. Пару $(C, G)$ часто называют конденсатором, имея в виду, что $G \backslash C$ является полем конденсатора, а составляющие его границу $\partial(G \backslash C)$ множества $\partial G$ и $\partial C$ являются обкладками этого конденсатора.

Конформной емкостью конденсатора $(C, G)$ называется величина

$$
\operatorname{cap}(C, G)=\inf \int_{G}|\nabla f|^{n} d v,
$$

где нижняя грань берется по всем гладким финитным в $G$ функциям, таким, что $f \equiv 1$ на $C$ и $0 \leqslant f \leqslant 1$ в $G$. Ясно, что значение емкости не изменится, если нижнюю грань здесь брать по более широкому множеству тех функций $f$ соболевского пространства $\stackrel{\circ}{L}_{n}^{1}(G)$, для которых $\left.f\right|_{C}=1$ в пространстве $L_{n}^{1}(G)$.

Произвольную риманову метрику $\tilde{g}$ на $M$ называют конформно эквивалентной или конформной метрике $g$, если $\tilde{g}=\lambda^{2} g$ на $M$, где $\lambda$ - некоторая положительная регулярная функция на $M$. Легко видеть (и это хорошо известно), что величина $\operatorname{cap}(C, G)$ инвариантна относительно конформных замен исходной метрики многообразия (см., например, [1]).

С помощью емкости можно конформно-инвариантно охарактеризовать массивность абсолюта - идеальной границы многообразия. Для данного многообразия $M$ величина $\operatorname{cap}(C, M)$ либо положительна, либо равна нулю независимо от выбора невырожденного континуума $C$.

Таким образом, указанное свойство обращения или необращения в нуль емкости $\operatorname{cap}(C, M)$ определяется только геометрией многообразия $M$ на «бесконечности», и в этом смысле часто говорят о нулевой или положительной конформной емкости абсолюта многообразия. Например, для евклидова пространства $\operatorname{cap}\left(C, \mathbb{R}^{n}\right)=0$, а для пространства Лобачевского $\operatorname{cap}\left(C, \mathbb{H}^{n}\right)>0$.

Многообразие $M$ будем называть многообразием конформно-параболического muna, если конформная емкость его абсолюта равна нулю, т. е. $\operatorname{cap}(C, M)=0$ для любого невырожденного континуума $C \subset M$; если же эта емкость положительна, то будем называть $M$ многообразием конформно-гиперболического типа.

Такое разделение многообразий по указанным конформным типам, очевидно, инвариантно относительно конформных замен римановой метрики многообразия.

\section{§2. Формулировка основных результатов}

Далее рассматривается произвольное $n$-мерное некомпактное риманово многообразие $M$ без края с некоторой исходной метрикой $g$. При переходе от метрики $g$ к конформной ей метрике $\tilde{g}$ одноименные геометрические объекты будут наделяться тильдой.

Теорема. Риманово многообразие $(M, g)$ имеет конформно-гиперболический тип тогда и только тогда, когда существует конформно эквивалентная метрике g риманова метрика $\tilde{g}$ бесконечного объема, в которой выполняется изопериметрическое неравенство

$$
\widetilde{V}(D) \leqslant \widetilde{S}(\partial D)
$$


для всех регулярных областей $D \subset M$, объем $\widetilde{V}(D)$ которых больше фиксированного (произвольно малого) положительного числа.

Здесь под регулярной областью понимается, как обычно, предкомпактная в $M$ область с кусочно-гладкой границей.

Таким образом, в конформном классе метрики риманова многообразия конформно-гиперболического типа изопериметрическая функция приводится к тому же линейному виду $P(x)=x$, что и в пространстве Лобачевского.

По поводу полноты метрики $\tilde{g}$ см. $\$ 4$.

Дополнение 1. Указанное в теореме неравенство $(*)$ является асимптотически точным в том смысле, что отношение $\widetilde{S}(\partial D) / \widetilde{V}(D)$ может быть сколь угодно близким к единице для регулярных областей сколь угодно большого $\tilde{g}$-объема.

Точнее, для любого $\varepsilon>0$ метрику $\tilde{g}$ можно подобрать так, что $1 \leqslant \widetilde{S}(\partial D) / \widetilde{V}(D)$ $\leqslant 1+\varepsilon$ для некоторой последовательности областей $D$, исчерпывающих $M$.

ДоПолнениЕ 2. Указанную в теореме метрику $\tilde{g}$ можно выбрать так, ито объем шара радиуса $r$ в этой метрике имеет порядок величины $e^{r}$ при $r \rightarrow$ $+\infty$.

Напомним [5], что изопериметрической размерностью многообразия бесконечного объема называется точная верхняя грань всех чисел $m>0$, для каждого из которых функция $P(x)=x^{(m-1) / m}$ (при всех $x>\varepsilon>0$ ) является изопериметрической функцией многообразия. Точную верхнюю грань изопериметрических размерностей многообразия по всем метрикам, конформным исходной метрике многообразия, будем называть (см. [6]) конформной изопериметрической размерностью многообразия.

Используя сформулированную выше теорему, можно получить

СледствиЕ. Риманово многообразие $M$ имеет конформно-гиперболический тип тогда и только тогда, когда его конформная изопериметрическая размерность равна $+\infty$.

\section{§3. Доказательства}

В этом параграфе мы изложим доказательства сформулированных утверждений.

ДоКАЗАТЕЛЬСТвО ТЕОРЕМЫ. Сначала о конформной гиперболичности риманова многообразия $M$, на котором выполняется изопериметрическое неравенство $(*)$. Это утверждение можно считать хорошо известным. Для полного многообразия $M$ оно получается с помощью леммы Альфорса-Громова [5] (см. $[1,3])$.

Прямое доказательство в общем случае вытекает, например, из следующей полезной оценки конформной емкости (см. $[7,19,20]$ или $[1,2])$ :

$$
\left(\int_{v(a)}^{v(r)} \widetilde{P}^{n /(1-n)}\right)^{1-n} \leqslant \operatorname{cap}\left(D_{a}, D_{r}\right),
$$

где $\left\{D_{r} \mid r \in \mathbb{R}_{+}\right\}$- концентрические компактные $g$-шары, $r$ - радиус шара $D_{r}, v(r)=\widetilde{V}\left(D_{r}\right)$ и $\widetilde{P}-$ изопериметрическая функция многообразия относительно конформно эквивалентной метрике $g$ римановой метрики $\tilde{g}$. Метрику $g$ можно считать полной, так как этого всегда можно добиться конформной заменой метрики. 
Тогда если в некоторой метрике $\tilde{g}$ бесконечного объема, конформной исходной метрике $g$, выполняется изопериметрическое неравенство $(*)$, то функция $\tilde{P}$ имеет вид $\widetilde{P}(x)=x$ при $x>\varepsilon>0$. Поэтому интеграл в (1) сходится при $r \rightarrow+\infty$. Следовательно, многообразие $M$ имеет конформно-гиперболический тип (так как $\operatorname{cap}\left(D_{a}, D_{r}\right) \searrow \operatorname{cap}\left(D_{a}, M\right)$ при $\left.r \nearrow+\infty\right)$. Попутно отметим, что в этом рассуждении участвовали только неравенства $(*)$ и $(1)$, т. е. если на многообразии выполнено изопериметрическое неравенство $(*)$, то без каких-либо дополнительных требований к метрике $\tilde{g}$ (например, бесконечности объема) уже можно сказать, что многообразие $M$ имеет конформно-гиперболический тип.

Перейдем теперь к основному утверждению теоремы о приведении изопериметрической функции к линейному каноническому виду на конформно-гиперболическом многообразии $M$.

Возьмем в $M$ компакт $C$ положительной емкости, но нулевого $g$-объема. В силу конформной гиперболичности многообразия $M$ существует (и единственна) экстремальная функция $u \in \stackrel{\circ}{L}_{n}^{1}(M),\left.u\right|_{C}=1$, такая, что

$$
\operatorname{cap}(C, M)=\int_{M}|\nabla u|^{n} d v>0 .
$$

При этом функция $u$ как обобщенная функция удовлетворяет в области $M \backslash C$ уравнению

$$
\Delta_{n} u \equiv \operatorname{div}\left(|\nabla u|^{n-2} \nabla u\right)=0,
$$

являющемуся уравнением Эйлера-Лагранжа рассмотренной вариационной задачи.

Изучению свойств решений уравнения (2), называемых $n$-гармоническими функциями, посвящено много работ (см., например, [8,9], а также книгу [10] и библиографию к ней), в которых классические свойства гармонических функций (принцип экстремума, неравенство и теоремы Гарнака и т. д.) перенесены на общий нелинейный случай. В частности, известно, что $n$-гармонические функции являются гладкими класса $C^{1, \alpha}$, причем они принадлежат классу $C^{\infty}$ там, где $\nabla u \neq 0$.

В силу строгого принципа экстремума для $n$-гармонических функций функция $u$ удовлетворяет неравенству $0<u<1$ всюду в $M \backslash C$. При этом, очевидно, $\inf u=0$ на $M$.

Имея функцию $u$, зададим теперь конформно эквивалентную метрике $g$ риманову метрику $\tilde{g}$ следующим образом:

$$
\tilde{g}=\left((n-1) \frac{|\nabla u|}{u}\right)^{2} g .
$$

Проверим, что изопериметрическое неравенство $(*)$ в метрике $\tilde{g}$ выполняется для произвольной регулярной области $D \subset M$.

Будем считать компакт $C$ гладкой гиперповерхностью в $M$, диффеоморфной $(n-1)$-мерному замкнутому диску (шару). Тогда к множеству $U=D \backslash C$ можно применить формулу Стокса, на основании которой и с учетом уравнения (2) имеем

$$
-\int_{\partial U}|\nabla u|^{n-2} \frac{\langle\nabla u, \nu\rangle}{u^{n-1}} d \sigma=(n-1) \int_{U} \frac{|\nabla u|^{n}}{u^{n}} d v,
$$


где $\nu$ - единичный вектор нормали к $\partial U$ (внешней по отношению к соответствующей компоненте $U)$, а $d \sigma-$ элемент $(n-1)$-меры Хаусдорфа границы $\partial U$.

Заметим, что граница $\partial U$ множества $U$ есть объединение множеств $C \cap \bar{D}$ и $\partial D \backslash C$, на первом из которых $\langle\nabla u, \nu\rangle \geqslant 0$. Поэтому левая часть формулы (4) не уменьшится, если интеграл в ней взять только по множеству $\partial D \backslash C$. А интеграл в правой части формулы (4) можно распространить на все множество $D$ ввиду того, что $V(C)=0$. Принимая во внимание сказанное, из (4) получаем неравенство

$$
(n-1) \int_{D}\left(\frac{|\nabla u|}{u}\right)^{n} d v \leqslant \int_{\partial D}\left(\frac{|\nabla u|}{u}\right)^{n-1} d \sigma
$$

которое и совпадает с изопериметрическим неравенством $(*)$ в метрике $\tilde{g}$, определенной формулой (3).

То, что $\widetilde{V}(M)=\infty$, мы увидим при доказательстве дополнений 1,2 .

ЗАмечАниЕ. Таким образом, мы доказали, что в метрике $\tilde{g}$ неравенство $(*)$ выполняется для всех регулярных областей, а не только для областей, объемы которых отграничены от нуля некоторой константой.

Однако мы не можем гарантировать, что построенная метрика $\tilde{g}$ всюду регулярна и невырожденна. Эти свойства метрики нарушаются (помимо множества $C$ ) в критических точках функции $u$, но вопрос о том, каково множество критических точек $n$-гармонической функции, при $n>2$ пока (как нам сообщили специалисты) остается открытым.

Чтобы обойти указанные трудности, мы добавим к построенной метрике $\tilde{g}$ положительный довесок (сколь угодно мало меняющий объем всего многообразия) так, чтобы получить регулярную нигде не вырожденную метрику. Тогда, используя уже доказанное в старой «метрике» неравенство $(*)$, в новой метрике приходим к неравенству

$$
\widetilde{V}(D)<\widetilde{S}(\partial D)+\varepsilon,
$$

справедливому для всех регулярных областей $D$.

Заметим теперь, что от метрики, в которой выполняется неравенство $(* *)$, можно умножением на число перейти к метрике, в которой справедливо неравенство $(*)$ для всех областей, объемы которых отграничены от нуля. При этом указанное число можно сделать сколь угодно близким к единице.

Действительно, пусть $m-$ произвольное натуральное число. Тогда если $\widetilde{V}(D)$ $>m \varepsilon$, то для таких областей из $(* *)$ имеем неравенство $a_{m} \widetilde{V}(D)<\widetilde{S}(\partial D)$, где $a_{m}=(m-1) / m$. Отсюда после умножения метрики на число $a_{m}^{2}$, которое можно выбрать сколь угодно близким к единице, получаем изопериметрическое неравенство $(*)$, справедливое для всех областей, объемы которых в построенной регулярной конформной исходной метрике больше положительной постоянной $m \varepsilon$. Как видно из приведенного построения, эта постоянная может быть выбрана сколь угодно близкой к нулю.

ДокАЗАТЕЛЬСтво ДоПОЛНЕНИЯ 1. Рассмотрим семейство областей $\left\{D_{t}, 0<t<1\right\}$, где $D_{t}=\{x \in M \mid u>t\}$. Области $\left\{D_{t}\right\}$ исчерпывают многообразие $M$ при $t \rightarrow 0$. Поскольку

$$
\widetilde{S}\left(\partial D_{t}\right)=-\int_{\partial D_{t}}|\nabla u|^{n-2} \frac{\langle\nabla u, \nu\rangle}{u^{n-1}} d \sigma
$$


формула (4) для множества $U=D_{t} \backslash C$ примет вид

$$
\widetilde{V}\left(D_{t}\right)=\widetilde{S}\left(\partial D_{t}\right)-\widetilde{S}(C) .
$$

Вместе с тем $\widetilde{S}\left(\partial D_{t}\right)=I / t^{n-1}$, где

$$
I=-\int_{\partial D_{t}}|\nabla u|^{n-2}\langle\nabla u, \nu\rangle d \sigma,
$$

причем ввиду уравнения (2) интеграл $I$ (поток) не зависит от $t$. Следовательно, $\widetilde{S}\left(\partial D_{t}\right) \rightarrow \infty$ при $t \rightarrow 0$.

Но тогда на основании формулы (5) многообразие $(M, \tilde{g})$ имеет бесконечный объем и изопериметрическое неравенство $(*)$ является асимптотически точным.

Заметим, что граница $\partial D_{t}$ области $D_{t}$ может быть негладкой только в критических точках функции $u$. Но по известному (см. [11, с. 104]) дополнению к теореме Сарда для почти всех $t \in(0,1)$ множество критических точек функции $u \in C^{1}$, лежащих на $\partial D_{t}$, имеет $(n-1)$-меру Хаусдорфа, равную нулю.

Поэтому можно проварьировать границу $\partial D_{t}$ вблизи указанных точек таким образом, чтобы получить область с кусочно-гладкой границей, но при этом изменить величины $\widetilde{V}\left(D_{t}\right)$ и $\widetilde{S}\left(\partial D_{t}\right)$ сколь угодно мало. Тогда ввиду $(5)$ асимптотическая точность изопериметрического неравенства $(*)$ будет реализована на семействе областей с гладкими границами.

В проведенном рассуждении мы применили формулу Стокса к области $D_{t}=$ $\{x \in M \mid u>t\}$, которая, вообще говоря, может и не быть предкомпактной. Разумеется, возможность такого применения формулы Стокса требует обоснования, и оно будет проведено нами для произвольной области $D$, имеющей конечный $n$-мерный объем $\widetilde{V}(D)$ и конечную $(n-1)$-меру Хаусдорфа (площадь) $\widetilde{S}(\partial D)$ границы области. Но, чтобы не нарушать основную нить изложения, мы вынесли рассмотрение указанного общего случая в $\$ 5$, посвященный обобщенной форме основной теоремы.

Отметим здесь только, что конечность $\tilde{g}$-объема области $D_{t}$ вытекает из очевидного неравенства

$$
\widetilde{V}\left(D_{t}\right)<(n-1)^{n} \frac{\operatorname{cap}(C, M)}{t^{n}},
$$

а так как в силу общей формулы коплощади (см., например, [11] или [12])

$$
\widetilde{V}\left(D_{t}\right)=(n-1) \int_{t}^{1} \frac{\widetilde{S}\left(\partial D_{\tau}\right)}{\tau} d \tau,
$$

то также конечна (для почти всех $t \in(0,1))$ и $\tilde{g}$-площадь границы области $D_{t}$.

ЗАмЕЧАНИЕ. Мы пока доказали дополнение 1 для метрики $\tilde{g}$, которая не вполне регулярна. Выделяя из семейства $\left\{D_{t}\right\}$ какую-либо исчерпывающую многообразие $M$ последовательность областей, например $\left\{D_{k^{-1}}, k=1,2, \ldots\right\}$, и следуя описанному в предыдущем замечании способу регуляризации метрики, можно изменить метрику $\tilde{g}$ так, чтобы значения величин $\widetilde{S}\left(\partial D_{k^{-1}}\right)$ и $\widetilde{V}\left(D_{k^{-1}}\right)$ при переходе к регулярной метрике изменились сколь угодно мало. Тогда в силу (5) отношение этих величин в новой метрике стремится к единице при $k \rightarrow \infty$.

Осталось заметить следующее. После проведенной регуляризации в новой метрике выполняется пока неравенство (**). Но, как указано в предыдущем замечании, путем умножения метрики на число, сколь угодно близкое к единице, можно 
получить метрику, в которой уже выполняется неравенство $(*)$. При этом отношение величин $\widetilde{S}\left(\partial D_{k^{-1}}\right)$ и $\widetilde{V}\left(D_{k^{-1}}\right)$ изменится сколь угодно мало, и, следовательно (ввиду (5)), оно будет сколь угодно близким к единице. Таким образом, для построенной регулярной метрики имеется асимптотическая точность неравенства $(*)$ в указанном выше смысле.

ДоКАЗАТЕЛЬСтвО доПОЛНЕНИЯ 2. Учитывая, что при рассмотренном выше способе регуляризации метрики $\tilde{g}$ можно считать, что объем многообразия меняется сколь угодно мало и относительное изменение больших расстояний тоже мало, нам достаточно провести доказательство дополнения 2 только в метрике $\tilde{g}$, определенной в (3).

Обратимся к рассмотренному при доказательстве дополнения 1 семейству областей $\left\{D_{t}\right\}$ и найдем сначала асимптотику роста объемов $\widetilde{V}\left(D_{t}\right)$ при $t \rightarrow 0$.

В силу формулы (6)

$$
\widetilde{V}^{\prime}\left(D_{t}\right)=-(n-1) \frac{\widetilde{S}\left(\partial D_{t}\right)}{t}
$$

что ввиду равенства (5) приводит к дифференциальному уравнению

$$
\widetilde{V}^{\prime}\left(D_{t}\right)=-(n-1) \frac{\widetilde{V}\left(D_{t}\right)+c}{t},
$$

где $c=\widetilde{S}(C)$. Интегрируя это уравнение, находим, что

$$
\ln \frac{\widetilde{V}\left(D_{t}\right)+c}{c}=(n-1)|\ln t| .
$$

Используя определение метрики $\tilde{g}$, нетрудно видеть, что правая часть последнего равенства совпадает с $\tilde{g}$-расстоянием $r=r(t)$ от множества $\partial D_{t}$ до $C$.

Действительно, $\tilde{g}$-длина любой кривой, соединяющей уровни $u_{1}$ и $u_{2}$ функции $u$, не меньше $(n-1)\left|\ln \left(u_{2} / u_{1}\right)\right|$ - модуля интеграла от производной функции $(n-1) \ln u$ вдоль этой кривой.

С другой стороны, длина любой интегральной кривой поля $\nabla \ln u$, соединяющей указанные уровни, равна $(n-1)\left|\ln \left(u_{2} / u_{1}\right)\right|$.

Тот факт, что такие соединяющие кривые действительно имеются, следует из того, что множество, где $\nabla u=0$, не разделяет уровни функции $u$ (иначе поток поля $\nabla u$ через любой уровень функции $u$ и емкость сар $(C, M)$ были бы равны нулю).

Итак, имеем асимптотику

$$
\widetilde{V}\left(D_{t}\right) \sim c e^{r} \quad \text { при } t \rightarrow 0 .
$$

Заметим теперь, что для $\tilde{g}$-геодезического шара $B(r)$ радиуса $r$ с центром в некоторой точке множества $C$ имеют место следующие включения:

$$
B(r) \subset D_{t(r)} \subset B(r+d),
$$

где постоянная $d$ равна $\tilde{g}$-диаметру множества $C$. Отсюда ввиду полученной выше асимптотики для $\widetilde{V}\left(D_{t}\right)$ заключаем, что

$$
c e^{r-d} \leqslant \widetilde{V}(B(r)) \leqslant c e^{r}
$$

при достаточно больших $r$, т. е. $\widetilde{V}(B(r)) \asymp e^{r}$ при $r \rightarrow \infty$. 
ДоКАЗАТЕЛЬСТВО СЛЕДстВИЯ. Прежде всего, в силу доказанной теоремы конформная изопериметрическая размерность конформно-гиперболического многообразия равна $+\infty$.

Обратное утверждение легко вытекает из уже приведенной оценки (1) конформной емкости. В самом деле, если в некоторой конформной исходной метрике $\tilde{g}$ многообразие $M$ (бесконечного объема) имеет изопериметрическую функцию вида $P(x)=x^{(m-1) / m}, x>\varepsilon$, для какого-либо числа $m>n$, то в приведенной выше оценке емкости интеграл сходится при $r \rightarrow+\infty$. Следовательно, многообразие $M$ имеет конформно-гиперболический тип.

ЗАмЕЧАНИЕ. Следуя совету рецензента, отметим, что проведенное доказательство устанавливает эквивалентность следующих свойств риманова многообразия $\left(M^{n}, g\right)$ : конформная изопериметрическая размерность многообразия больше $n$; она равна бесконечности; многообразие имеет конформно-гиперболический тип.

\section{§4. Комментарий}

Мы уже затронули в предыдущих замечаниях вопрос о возможных особенностях построенной метрики $\tilde{g}$ и об одном из способов ее регуляризации.

Отметим еще, что метрика $\tilde{g}$ может оказаться неполной. Например, это заведомо так, если многообразие имеет точечный прокол или, точнее, точечный элемент границы. Дело в том, что удаление из многообразия $M$ точки или вообще множества емкости нуль не меняет функцию $u$, участвующую в определении метрики $\tilde{g}$ (формула (3)).

Однако метрическое пополнение пространства $(M, \tilde{g})$ присоединяет к $M$ малое множество случайных параболических граничных элементов (типа выколотых точек), превращая $M$ во вполне гиперболическое некомпактное пространство $\bar{M}$ с полной метрикой $\bar{g}$ на нем. При этом изопериметрическое неравенство (*) будет выполняться уже на полном пространстве $(\bar{M}, \bar{g})$ и для всех областей $D$ в $\bar{M}$, которые имеют в $(\bar{M}, \bar{g})$ конечный объем и конечную площадь границы.

Это, в частности, означает, что теорема остается справедливой, даже если в определении регулярной области отказаться от условия предкомпактности. Доказательство такого обобщения теоремы и одновременно завершение доказательства дополнения 1 читатель найдет в следующем параграфе.

\section{§5. Общая форма теоремы}

Здесь мы покажем, что для многообразия $(M, g)$ конформно-гиперболического типа изопериметрическое неравенство (*) в определенной формулой (3) римановой метрике $\tilde{g}$ справедливо для произвольной (не обязательно предкомпактной) области $D$ конечного объема $\widetilde{V}(D)$.

Вопросы, связанные с регулярностью и невырожденностью искомой метрики, в этом случае решаются так же, как и выше, и мы на них уже не будем останавливаться.

Назовем для краткости область $D$ допустимой относительно метрики $\tilde{g}$, если она имеет в указанной метрике конечный $n$-мерный объем $\widetilde{V}(D)$ и конечную $(n-1)$-меру Хаусдорфа $\widetilde{S}(\partial D)$ границы $\partial D$.

В основе распространения изопериметрического неравенства $(*)$ с регулярных (предкомпактных) областей на произвольные допустимые области лежат две приведенные ниже леммы. 
Прежде чем формулировать первую из них, напомним, что если $K$ - компакт, лежащий в замкнутой не обязательно компактной области $\bar{D} \subset M$, то, как и в случае открытой области $D$, конформная емкость конденсатора $(K, \bar{D})$, $\operatorname{cap}(K, \bar{D})$, определяется соотношением

$$
\operatorname{cap}(K, \bar{D})=\inf \int_{M}|\nabla f|^{n} d v,
$$

где нижняя грань берется по всем гладким финитным в $\bar{D}$ функциям $f$ на $M$, таким, что $f \equiv 1$ на $K$ и $0 \leqslant f \leqslant 1$ в $\bar{D}$. Понятно, что значение емкости не изменится, если нижнюю грань здесь брать по более широкому множеству всех функций $f$ соболевского пространства $\stackrel{\circ}{L}_{n}^{1}(\bar{D})$, для которых $\left.f\right|_{K}=1$ в пространстве $L_{n}^{1}(\bar{D})$.

Стоит отметить, что при рассмотрении емкости $\operatorname{cap}(K, \bar{D})$ класс допустимых функций шире, чем в случае емкости $\operatorname{cap}(K, D)$. Например, если $\bar{D}-$ компакт, то функция $f \equiv 1$ является допустимой при вычислении $\operatorname{cap}(K, \bar{D})$; поэтому $\operatorname{cap}(K, \bar{D})=0$ для любой предкомпактной области $D$, хотя, возможно, $\operatorname{cap}(K, D)>0$.

Вместе с тем, если $\bar{D}$ не является компактом, то на многообразии конформногиперболического типа, вообще говоря, $\operatorname{cap}(K, \bar{D})>0$ именно за счет выхода на абсолют.

Переходим к леммам. Всюду ниже $D$ - область на многообразии $(M, g)$ конформно-гиперболического типа, а $\tilde{g}$ - метрика, определенная формулой $(3)$.

Лемма 1. Пусть $D$ - произвольная область конечного $\tilde{g}$-объема $\tilde{V}(D)$. Tогда

$$
\operatorname{cap}(K, \bar{D})=0 \text {. }
$$

Для доказательства леммы предположим сначала, что

$$
\inf _{D} u=\delta>0
$$

где $u$ - экстремальная функция из формулы (3), определяющей метрику $\tilde{g}$.

Тогда функция $f=\min \{u / \delta, 1\} \equiv 1$ принадлежит, очевидно, пространству $\stackrel{\circ}{L}_{n}^{1}(\bar{D})$ и, следовательно, является допустимой для емкости $\operatorname{cap}(K, \bar{D})$. Отсюда сразу получаем (7).

Предположим теперь, что условие (8) не выполняется, т. е. существует некоторая последовательность точек $x_{k} \in D$, вдоль которой $t_{k}=u\left(x_{k}\right) \rightarrow 0$. При этом, не ограничивая общности, можно считать числовую последовательность $\left\{t_{k}\right\}$ строго убывающей (стремящейся к нулю). Зафиксируем значение $t_{0}$, такое, что $\min _{K} u>t_{0}$, и для каждого значения $t_{k}$, меньшего, чем $t_{0}$, определим функцию $f_{k}$, полагая

$$
f_{k}=\max \left\{\frac{\ln \left(u / t_{k}\right)}{\ln \left(t_{0} / t_{k}\right)}, 0\right\} .
$$

Легко видеть, что функция $f_{k}$ является допустимой для $\operatorname{cap}(K, \bar{D})$, и так как

$$
\int_{D}\left|\nabla f_{k}\right|^{n} d v \leqslant \frac{\int_{D}(|\nabla u| / u)^{n} d v}{\ln ^{n}\left(t_{0} / t_{k}\right)}
$$


то получаем неравенство

$$
\operatorname{cap}(K, \bar{D}) \leqslant \frac{\tilde{V}(D)}{(n-1)^{n} \ln ^{n}\left(t_{0} / t_{k}\right)}
$$

Отсюда, устремляя последовательность $\left\{t_{k}\right\}$ к нулю, приходим к равенству $(7)$. Лемма 1 доказана.

Введем используемое в дальнейшем обозначение

$$
F_{u}(\Gamma)=\int_{\Gamma}|\nabla u|^{n-2} \frac{\langle\nabla u, \nu\rangle}{u^{n-1}} d s
$$

для любой $(n-1)$-мерной кусочно-гладкой поверхности $\Gamma$, ориентированной полем нормалей $\nu$. Очевидно, что $(n-1)^{n-1}\left|F_{u}(\Gamma)\right| \leqslant \widetilde{S}(\Gamma)$.

Лемма 2. Пусть D- произвольная допустимая относительно метрики $\tilde{g}$ область. Тогда имеет место неравенство

$$
(n-1)^{n-1}\left|F_{u}(\partial B \cap \bar{D})\right| \leqslant \widetilde{V}(D \backslash B)+\widetilde{S}(\partial D \backslash B)
$$

для любого регулярного компакта $B$, содержащего компакт $C$.

Возьмем произвольную допустимую для $\operatorname{cap}(B \cap \bar{D}, \bar{D})$ функцию $f$, которую, не ограничивая общности, можно считать гладкой в $\bar{D}$, обращающейся в нуль вне некоторой регулярной предкомпактной области $G$, содержащей $B$ и такой, что $f \equiv 1$ на $B \cap \bar{D}$ и $0 \leqslant f \leqslant 1$ всюду в $\bar{D}$. Применяя затем к области $U=(G \backslash B) \cap D$ формулу Стокса, получаем

$-\int_{\partial U} f|\nabla u|^{n-2} \frac{\langle\nabla u, \nu\rangle}{u^{n-1}} d s=-\int_{U}|\nabla u|^{n-2} \frac{\langle\nabla f, \nabla u\rangle}{u^{n-1}} d v+(n-1) \int_{U} f(|\nabla u| / u)^{n} d v$.

При этом учтено, согласно $(2)$, что $\Delta_{n} u=0$ всюду в $U$.

Граница $\partial U$ состоит из частей границ $\partial B, \partial G, \partial D$. На первой из них $f \equiv 1$, а на второй $f \equiv 0$. Значит, приведенная выше формула дает неравенство

$$
\begin{aligned}
& \left|F_{u}(\partial B \cap \bar{D})\right| \leqslant \int_{U}|\nabla u|^{n-2} \frac{|\langle\nabla f, \nabla u\rangle|}{u^{n-1}} d v \\
& \quad+(n-1) \int_{U}(|\nabla u| / u)^{n} d v+\int_{\partial D \cap \partial U}(|\nabla u| / u)^{n-1} d s .
\end{aligned}
$$

Применяя здесь к первому из интегралов, стоящих в правой части, неравенство Гёльдера, получаем

$(n-1)^{n-1}\left|F_{u}(\partial B \cap \bar{D})\right| \leqslant \widetilde{V}(U)+\widetilde{S}(\partial D \cap \partial U)+\left(\int_{U}|\nabla f|^{n} d v\right)^{1 / n}(\widetilde{V}(U))^{(n-1) / n}$.

Отсюда, ввиду произвольности допустимой для $\operatorname{cap}(B \cap \bar{D}, \bar{D})$ функции $f$, приходим к неравенству

$(n-1)^{n-1}\left|F_{u}(\partial B \cap \bar{D})\right| \leqslant \widetilde{V}(D \backslash B)+\widetilde{S}(\partial D \backslash B)+(\operatorname{cap}(B \cap \bar{D}, \bar{D}))^{1 / n}(\widetilde{V}(D \backslash B))^{(n-1) / n}$.

Наконец, применяя к последнему неравенству лемму 1, приходим к искомому неравенству (9). Лемма 2 доказана. 
Теперь мы в состоянии доказать изопериметрическое неравенство $(*)$ для произвольной допустимой относительно метрики $\tilde{g}$ области $D$.

Рассмотрим произвольное исчерпание многообразия $M$ регулярными областями $B_{k}, k \in \mathbb{N}$, и для каждого $k$ применим к предкомпактной области $U=$ $\left(B_{k} \backslash C\right) \cap D$ формулу Стокса (4). Поскольку граница $\partial U$ состоит из следующих частей:

$$
\Gamma_{C}=C \cap \bar{D}, \quad \Gamma_{B}=\partial B_{k} \cap \bar{D}, \quad \Gamma_{U}=\partial U \backslash\left(\Gamma_{C} \cup \Gamma_{B}\right),
$$

то формулу (4) можно переписать таким образом:

$$
\widetilde{V}(U)=-(n-1)^{n-1}\left(F_{u}\left(\Gamma_{C}\right)+F_{u}\left(\Gamma_{B}\right)+F_{u}\left(\Gamma_{U}\right)\right) .
$$

Так как очевидно, что $F_{u}\left(\Gamma_{C}\right) \geqslant 0$ (ибо $\langle\nabla u, \nu\rangle \geqslant 0$ на $C$ ), то из (10) имеем неравенство

$$
\widetilde{V}(U) \leqslant(n-1)^{n-1}\left|F_{u}\left(\Gamma_{B}\right)\right|+\widetilde{S}(\partial D) .
$$

Пусть теперь $k \rightarrow+\infty$. Тогда ввиду допустимости области $D$

$$
\widetilde{V}\left(D \backslash B_{k}\right) \rightarrow 0, \quad \widetilde{S}\left(\partial D \backslash B_{k}\right) \rightarrow 0 ;
$$

следовательно, в силу леммы 2

$$
\left|F_{u}\left(\Gamma_{B}\right)\right|=\left|F_{u}\left(\partial B_{k} \cap \bar{D}\right)\right| \rightarrow 0 .
$$

С учетом этого, переходя к пределу в неравенстве (11), получаем изопериметрическое неравенство $(*)$.

\section{§6. Заключительные замечания}

Нашей целью было решение конкретной задачи. Мы не обсуждали здесь емкости, отличные от конформных, или иные понятия гиперболичности (параболичности) многообразий и соответствующие им признаки и критерии типа, возникающие в глобальном анализе (римановы поверхности, уравнения в частных производных на многообразиях, спектры операторов, динамика, диффузия, броуновское движение, ...). Много работ разных лет посвящено этим вопросам (см. например, [13-18] и недавний обзор [19] с обширной библиографией).

Наконец, возвращаясь к формулировке основной теоремы, заметим, что, повидимому, существует регулярная метрика, в которой изопериметрическое неравенство $(*)$ выполняется без каких-либо ограничений на объем области. Мы это уже показали для метрики, которая имела особенности там, где $\nabla u=0$. Полных сведений о множестве критических точек $n$-гармонической функции при $n>2$, как мы отмечали, пока нет. Однако на обсуждаемый вопрос, наверное, можно ответить и без этого ${ }^{1)}$.

Благодарности. Авторы выражают благодарность Российскому фонду фундаментальных исследований, при финансовой поддержке которого была выполнена эта работа. Первый автор благодарит также Институт высших научных исследований (IHES) во Франции и Исследовательский институт математики (FIMETH Zürich) в Швейцарии за гостеприимство во время выполнения этой работы. Авторы признательны рецензентам за конструктивные замечания; они учтены в окончательной редакции этой статьи.

1) Добавление при корректуре: вопрос положительно решен; доказательство будет опубликовано позже. 


\section{ЛИТЕРАТУРА}

1. Зорич B. A., Кесельман B. М. О конформном типе риманова многообразия. Функц. анализ и его прил., 30, вып. 2, 40-55 (1996).

2. Zorich V. A. Asymptotic geometry and conformal types of Carnot-Carathéodory spaces. Geom. Funct. Anal., 9, No. 2, 393-411 (1999).

3. Grimaldi $R$., Pansu $P$. Sur la croissance du volume dans une classe conforme. J. Math. Pures Appl., 9 (71), No. 1, 1-19 (1992).

4. Зорич B. A., Кесельман B. М. Канонический вид изопериметрического неравенства на многообразиях конформно-гиперболического типа. УМН, 54, вып. 3, 164-165 (1999).

5. Gromov M. with appendices by Katz M., Pansu P., and Semmes S. Metric structures for Riemannian and non-Riemannian spaces. Birkhäuser, Boston-Basel-Berlin, 1999.

6. Зорич B. A., Кесельман B. М. Конформный тип и изопериметрическая размерность риманова многообразия. Матем. заметки, 63, № 3, 379-385 (1998).

7. Мазья В. Г. Пространства С. Л. Соболева. Изд-во Ленинградского ун-та, Л., 1985.

8. Решетняк Ю. Г. Пространственные отображения с ограниченным искажением. Новосибирск, Наука, 1982.

9. Ладыженская О. А., Уральщева Н. Н. Линейные и квазилинейные уравнения эллиптического типа. Наука, М., 1973.

10. Heinonen J., Kilpelainen T., Martio $O$. Nonlinear potential theory of degenerate elliptic equations. Oxford University Press, 1993.

11. Бураго Ю. Д., Залгаллер В. А. Геометрические неравенства. Наука, Л., 1980.

12. Федерер $Г$. Геометрическая теория меры. Наука, М., 1987.

13. Ahlfors L. Sur le type d'une surface de Riemann. C. R. Acad. Sci. Paris, Ser. A, 201, 30-32 (1935).

14. Ahlfors L., Calabi E., Morse M., Sario L., Spencer D., eds. Contributions to the theory of Riemann surfaces, Centennial celebration of Riemann's dissertation. Ann. of Math. Stud., Vol. 30, Princeton University Press, Princeton, 1953.

15. Cheeger $J$. A lower bound for the smallest eigenvalue of the Laplacian. In: Problems in Analysis: A Symposium in Honor of Salomon Bochner, Princeton University Press, Princeton, 1970, pp. 195-199.

16. Cheng S. V., Yau S. T. Differential equations on Riemannian manifolds and their geometric applications. Comm. Pure Appl. Math., 28, 333-354 (1975).

17. Milnor J. On deciding whether a surface is parabolic or hyperbolic. Amer. Math. Monthly, 84, 1, 43-46 (1977).

18. Varopoulos N., Saloff-Coste L., Coulhon T. Analysis and geometry on groups. Cambridge University Press, Cambridge, 1992.

19. Grigor'yan A. Analytic and geometric background of recurrence and non-explosion of the Brownian motion. Bull. Amer. Math. Soc. (N.S.), 36, No. 2, 135-249 (1999).

20. Grigor'yan A. Isoperimetric inequality and capacities on Riemannian manifolds. In: Oper. Theory Adv. Appl., vol. 109, Birkhäuser Verlag, Basel, 1999, pp. 139-153.

Московский государственный университет, механико-математический факультет

Поступило в редакцию e-mail: vzor@online.ru

Московский государственный индустриальный университет, кафедра математики

e-mail: kvlm@online.ru 